\title{
Bayesian Classification With Gaussian Processes
}

\author{
Christopher K.I. Williams, Member, IEEE Computer Society, and David Barber
}

\begin{abstract}
We consider the problem of assigning an input vector to one of $m$ classes by predicting $P(c \mid x)$ for $c=1, \ldots, m$. For a twoclass problem, the probability of class one given $\boldsymbol{x}$ is estimated by $\sigma(y(\boldsymbol{x}))$, where $\sigma(y)=1 /\left(1+e^{-y}\right)$. A Gaussian process prior is placed on $y(\boldsymbol{x})$, and is combined with the training data to obtain predictions for new $\boldsymbol{x}$ points. We provide a Bayesian treatment, integrating over uncertainty in $y$ and in the parameters that control the Gaussian process prior; the necessary integration over $y$ is carried out using Laplace's approximation. The method is generalized to multiclass problems $(m>2)$ using the softmax function. We demonstrate the effectiveness of the method on a number of datasets.
\end{abstract}

Index Terms-Gaussian processes, classification problems, parameter uncertainty, Markov chain Monte Carlo, hybrid Monte Carlo, Bayesian classification.

\section{INTRODUCTION}

W E consider the problem of assigning an input vector $x$ to one out of $m$ classes by predicting $P(c \mid x)$ for $c=1$, $\ldots, m$. A classic example of this method is logistic regression. For a two-class problem, the probability of class 1 given $x$ is estimated by $\sigma\left(\boldsymbol{w}^{T} \boldsymbol{x}+b\right)$, where $\sigma(y)=1 /\left(1+e^{-y}\right)$. However, this method is not at all "flexible," i.e., the discriminant surface is simply a hyperplane in $x$-space. This problem can be overcome, to some extent, by expanding the input $x$ into a set of basis functions $\{\phi(x)\}$, for example quadratic functions of the components of $x$. For a highdimensional input space, there will be a large number of basis functions, each one with an associated parameter, and one risks "overfitting" the training data. This motivates a Bayesian treatment of the problem, where the priors on the parameters encourage smoothness in the model.

Putting priors on the parameters of the basis functions indirectly induces priors over the functions that can be produced by the model. However, it is possible (and we would argue, perhaps more natural) to put priors directly over the functions themselves. One advantage of functionspace priors is that they can impose a general smoothness constraint without being tied to a limited number of basis functions. In the regression case where the task is to predict a real-valued output, it is possible to carry out nonparametric regression using Gaussian Processes (GPs); see, e.g., [25], [28]. The solution for the regression problem under a GP prior (and Gaussian noise model) is to place a kernel function on each training data point, with coefficients determined by solving a linear system. If the parameters $\boldsymbol{\theta}$ that

- C.K.I. Williams is with the Department of Artificial Intelligence, University of Edinburgh, Edinburgh EH1 2QL, Scotland, UK.

E-mail:ckiw@dai.ed.ac.uk.

- D. Barber is with RWCP, Theoretical Foundation SNN, University of Nijmegen, 6525 EZ Nijmegen, The Netherlands.

E-mail: davidb@mbfys.kun.nl.

Manuscript received 1 Dec. 1997; revised 5 Oct. 1998. Recommended for acceptance by A. Webb.

For information on obtaining reprints of this article, please send e-mail to: tpami@computer.org, and reference IEEECS Log Number 108019. describe the Gaussian process are unknown, Bayesian inference can be carried out for them, as described in [28].

The Gaussian Process method can be extended to classification problems by defining a GP over $y$, the input to the sigmoid function. This idea has been used by a number of authors, although previous treatments typically do not take a fully Bayesian approach, ignoring uncertainty in both the posterior distribution of $y$ given the data, and uncertainty in the parameters $\boldsymbol{\theta}$. This paper attempts a fully Bayesian treatment of the problem, and also introduces a particular form of covariance function for the Gaussian process prior which, we believe, is useful from a modeling point of view.

The structure of the remainder of the paper is as follows: Section 2, discusses the use of Gaussian processes for regression problems, as this is essential background for the classification case. In Section 3, we describe the application of Gaussian processes to two-class classification problems, and extend this to multiple-class problems in Section 4. Experimental results are presented in Section 5 , followed by a discussion in Section 6. This paper is a revised and expanded version of [1].

\section{Gaussian Processes for Regression}

It will be useful to first consider the regression problem, i.e., the prediction of a real valued output $y_{*}=y\left(x_{*}\right)$ for a new input value $\boldsymbol{x}_{*}$, given a set of training data $\mathcal{D}=\left\{\left(x_{i}, t_{i}\right), i=1\right.$ ...n $n$. This is of relevance because our strategy will be to transform the classification problem into a regression problem by dealing with the input values to the logistic transfer function.

A stochastic process prior over functions allows us to specify, given a set of inputs, $x_{1}, \ldots x_{n}$, the distribution over their corresponding outputs $y \stackrel{\text { def }}{=}\left(y\left(x_{1}\right), y\left(x_{2}\right), \ldots, y\left(x_{n}\right)\right)$. We denote this prior over functions as $P(y)$, and similarly, $P\left(y_{*}, y\right)$ for the joint distribution including $y_{*}$. If we also specify $P(\boldsymbol{t} \mid \boldsymbol{y})$, the probability of observing the particular values 
$\boldsymbol{t}=\left(t_{1}, \ldots t_{n}\right)^{T}$ given actual values $\boldsymbol{y}$ (i.e., a noise model), then we have that

$$
\begin{aligned}
P\left(y_{*} \mid \boldsymbol{t}\right) & =\int P\left(y_{*}, \boldsymbol{y} \mid \boldsymbol{t}\right) d \boldsymbol{y} \\
& =\frac{1}{P(\boldsymbol{t})} \int P\left(y_{*} \mid \boldsymbol{y}\right) P(\boldsymbol{y}) P(\boldsymbol{t} \mid \boldsymbol{y}) d \boldsymbol{y} \\
& =\int P\left(y_{*} \mid \boldsymbol{y}\right) P(\boldsymbol{y} \mid \boldsymbol{t}) d \boldsymbol{y} .
\end{aligned}
$$

Hence, the predictive distribution for $y_{*}$ is found from the marginalization of the product of the prior and the noise model. Note that in order to make predictions, it is not necessary to deal directly with priors over function space, only $n$ - or $n+1$-dimensional joint densities. However, it is still not easy to carry out these calculations unless the densities involved have a special form.

If $P(\boldsymbol{t} \mid \boldsymbol{y})$ and $P\left(y_{*}, \boldsymbol{y}\right)$ are Gaussian, then $P\left(y_{*} \mid \boldsymbol{t}\right)$ is a Gaussian whose mean and variance can be calculated using matrix computations involving matrices of size $n \times n$. Specifying $P\left(y_{*}, y\right)$ to be a multidimensional Gaussian (for all values of $n$ and placements of the points $x_{*}, x_{1}, \ldots x_{n}$ ) means that the prior over functions is a Gaussian process. More formally, a stochastic process is a collection of random variables $\{Y(x) \mid x \in X\}$ indexed by a set $X$. In our case, $X$ will be the input space with dimension $d$, the number of inputs. A GP is a stochastic process which can be fully specified by its mean function $\mu(x)=E[Y(x)]$ and its covariance function $C\left(x, x^{\prime}\right)=E\left[(Y(x)-\mu(x))\left(Y\left(x^{\prime}\right)-\mu\left(x^{\prime}\right)\right)\right]$; any finite set of $Y$ variables will have a joint multivariate Gaussian distribution. Below we consider GPs which have $\mu(x) \equiv 0$.

If we further assume that the noise model $P(t \mid y)$ is Gaussian with mean zero and variance $\sigma^{2} I$, then the predicted mean and variance at $x_{*}$ are given by

$$
\begin{aligned}
\hat{y}\left(x_{*}\right) & =\boldsymbol{k}^{T}\left(x_{*}\right)\left(K+\sigma^{2} I\right)^{-1} t \\
\sigma_{\hat{y}}^{2}\left(x_{*}\right) & =C\left(x_{*}, x_{*}\right)-\boldsymbol{k}^{T}\left(x_{*}\right)\left(K+\sigma^{2} I\right)^{-1} k\left(x_{*}\right),
\end{aligned}
$$

where $[K]_{i j}=C\left(x_{i}, x_{j}\right)$ and $\boldsymbol{k}\left(x_{*}\right)=\left(C\left(x_{*}, x_{1}\right), \ldots, C\left(x_{*}, x_{n}\right)\right)^{T}$ (see, e.g., [25]).

\subsection{Parameterizing the Covariance Function}

There are many reasonable choices for the covariance function. Formally, we are required to specify functions which will generate a non-negative definite covariance matrix for any set of points $\left(x_{1}, \ldots, x_{k}\right)$. From a modeling point of view, we wish to specify covariances so that points with nearby inputs will give rise to similar predictions. We find that the following covariance function works well:

$$
C\left(x, x^{\prime}\right)=v_{0} \exp \left\{-\frac{1}{2} \sum_{l=1}^{d} w_{l}\left(x_{l}-x_{l}^{\prime}\right)^{2}\right\}+v_{1},
$$

where $x_{l}$ is the $l$ th component of $x$ and $\boldsymbol{\theta}=\left(\log v_{0}, \log v_{1}\right.$, $\left.\log w_{1}, \ldots, \log w_{d}\right)$ is the vector of parameters that are needed to define the covariance function. Note that $\boldsymbol{\theta}$ is analogous to the hyperparameters in a neural network. We define the parameters to be the log of the variables in (4) since these are positive scale-parameters. This covariance function can be obtained from a network of Gaussian radial basis functions in the limit of an infinite number of hidden units [27].

The $w_{l}$ parameters in (4) allow a different length scale on each input dimension. For irrelevant inputs, the corresponding $w_{l}$ will become small, and the model will ignore that input. This is closely related to the Automatic Relevance Determination (ARD) idea of MacKay [10] and Neal [15]. The $v_{0}$ variable specifies the overall scale of the prior. $v_{1}$ specifies the variance of a zero-mean offset which has a Gaussian distribution.

The Gaussian process framework allows quite a wide variety of priors over functions. For example, the OrnsteinUhlenbeck process (with covariance function $C\left(x, x^{\prime}\right)=$ $\left.e^{-\left|x-x^{\prime}\right|}\right)$ has very rough sample paths which are not meansquare differentiable. On the other hand, the squared exponential covariance function of (4) gives rise to an infinitely m.s. differentiable process. In general, we believe that the GP method is a quite general-purpose route for imposing prior beliefs about the desired amount of smoothness. For reasonably high-dimensional problems, this needs to be combined with other modeling assumptions such as ARD. Another modeling assumption that may be used is to build up the covariance function as a sum of covariance functions, each one of which may depend on only some of the input variables (see Section 3.3 for further details).

\subsection{Dealing With Parameters}

Given a covariance function, it is straightforward to make predictions for new test points. However, in practical situations we are unlikely to know which covariance function to use. One option is to choose a parametric family of covariance functions (with a parameter vector $\boldsymbol{\theta}$ ), and then either to estimate the parameters (for example, using the method of maximum likelihood) or to use a Bayesian approach where a posterior distribution over the parameters is obtained.

These calculations are facilitated by the fact that the log likelihood $l=\log P(\mathcal{D} \mid \boldsymbol{\theta})$ can be calculated analytically as

$$
l=-\frac{1}{2} \log |\widetilde{K}|-\frac{1}{2} t^{T} \widetilde{K}^{-1} t-\frac{n}{2} \log 2 \pi,
$$

where $\widetilde{K}=K+\sigma^{2} I$ and $|\widetilde{K}|$ denotes the determinant of $\widetilde{K}$. It is also possible to express analytically the partial derivatives of the log likelihood with respect to the parameters

$$
\frac{\partial l}{\partial \theta_{i}}=-\frac{1}{2} \operatorname{tr}\left(\widetilde{K}^{-1} \frac{\partial \widetilde{K}}{\partial \theta_{i}}\right)+\frac{1}{2} t^{T} \widetilde{K}^{-1} \frac{\partial \widetilde{K}}{\partial \theta_{i}} \widetilde{K}^{-1} t,
$$

(see, e.g., [11]).

Given $l$ and its derivatives with respect to $\boldsymbol{\theta}$, it is straightforward to feed this information to an optimization package in order to obtain a local maximum of the likelihood.

In general one may be concerned about making point estimates when the number of parameters is large relative to the number of data points, or if some of the parameters may be poorly determined, or if there may be local maxima in the likelihood surface. For these reasons, the Bayesian approach of defining a prior distribution over the parameters and then obtaining a posterior distribution once the data $\mathcal{D}$ has been seen is attractive. To make a prediction for a new test point $x_{*}$ one simply averages over the posterior distribution $P(\boldsymbol{\theta} \mid \mathcal{D})$, i.e., 

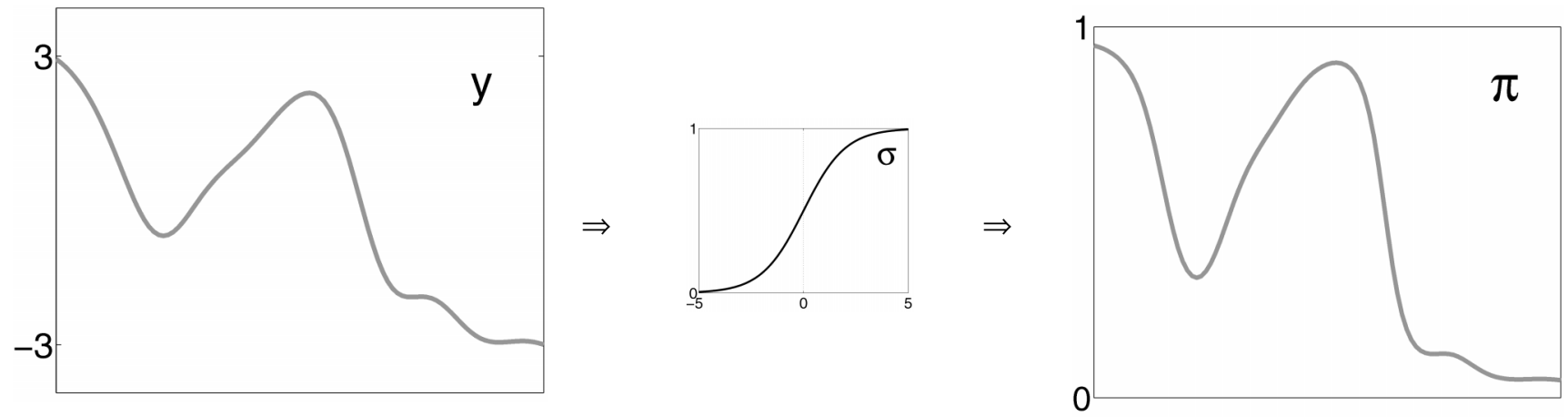

Fig. 1. $\pi(\boldsymbol{x})$ is obtained from $y(\boldsymbol{x})$ by "squashing" it through the sigmoid function $\sigma$.

$$
P\left(y_{*} \mid \mathcal{D}\right)=\int P\left(y_{*} \mid \boldsymbol{\theta}, \mathcal{D}\right) P(\boldsymbol{\theta} \mid \mathcal{D}) d \boldsymbol{\theta}
$$

For GPs, it is not possible to do this integration analytically in general, but numerical methods may be used. If $\boldsymbol{\theta}$ is of sufficiently low dimension, then techniques involving grids in $\boldsymbol{\theta}$-space can be used.

If $\boldsymbol{\theta}$ is high dimensional, it is very difficult to locate the regions of parameter space which have high-posterior density by gridding techniques or importance sampling. In this case, Markov chain Monte Carlo (MCMC) methods may be used. These work by constructing a Markov chain whose equilibrium distribution is the desired distribution $P(\boldsymbol{\theta} \mid \mathcal{D})$; the integral in (7) is then approximated using samples from the Markov chain.

Two standard methods for constructing MCMC methods are the Gibbs sampler and Metropolis-Hastings algorithms (see, e.g., [5]). However, the conditional parameter distributions are not amenable to Gibbs sampling if the covariance function has the form given by (4), and the MetropolisHastings algorithm does not utilize the derivative information that is available, which means that it tends to have an inefficient random-walk behavior in parameter-space. Following the work of Neal [15] on Bayesian treatment of neural networks, Williams and Rasmussen [28] and Rasmussen [17] have used the Hybrid Monte Carlo (HMC) method of Duane et al. [4] to obtain samples from $P(\boldsymbol{\theta} \mid \mathcal{D})$. The HMC algorithm is described in more detail in Appendix D.

\section{Gaussian Processes for Two-Class CLASSIFICATION}

For simplicity of exposition, we will first present our method as applied to two-class problems; the extension to multiple classes is covered in Section 4.

By using the logistic transfer function to produce an output which can be interpreted as $\pi(x)$, the probability of the input $x$ belonging to class 1 , the job of specifying a prior over functions $\pi$ can be transformed into that of specifying a prior over the input to the transfer function, which we shall call the activation, and denote by $y$ (see Fig. 1). For the two-class problem we can use the logistic function $\pi(x)=$ $\sigma(y(x))$, where $\sigma(y)=1 /\left(1+e^{-y}\right)$. We will denote the probability and activation corresponding to input $x_{i}$ by $\pi_{i}$ and $y_{i}$ respectively. Fundamentally, the GP approaches to classification and regression problems are similar, except that the error model which is $t \sim \mathrm{N}\left(y, \sigma^{2}\right)$ in the regression case is replaced by $t \sim \operatorname{Bern}(\sigma(y))$. The choice of $v_{0}$ in (4) will affect how "hard" the classification is; i.e., if $\pi(x)$ hovers around 0.5 or takes on the extreme values of 0 and 1 .

Previous and related work to this approach is discussed in Section 3.3.

As in the regression case, there are now two problems to address

1) making predictions with fixed parameters and

2) dealing with parameters.

We shall discuss these issues in turn.

\subsection{Making Predictions With Fixed Parameters}

To make predictions when using fixed parameters, we would like to compute $\hat{\pi}_{*}=\int \pi_{*} P\left(\pi_{*} \mid t\right) d \pi_{*}$, which requires us to find $P\left(\pi_{*} \mid \boldsymbol{t}\right)=P\left(\pi\left(\boldsymbol{x}_{*}\right) \mid \boldsymbol{t}\right)$ for a new input $\boldsymbol{x}_{*}$. This can be done by finding the distribution $P\left(y_{*} \mid t\right)$ ( $y_{*}$ is the activation of $\boldsymbol{\pi}_{*}$ ), and then using the appropriate Jacobian to transform the distribution. Formally, the equations for obtaining $P\left(y_{*} \mid t\right)$ are identical to (1), (2), and (3). However, even if we use a GP prior so that $P\left(y_{*}, y\right)$ is Gaussian, the usual expression for $P(t \mid y)=\prod \pi_{i}^{t_{i}}\left(1-\pi_{i}\right)^{1-t_{i}}$ for classification data (where the $t$ s take on values of zero or one) means that the marginalization to obtain $P\left(y_{*} \mid t\right)$ is no longer analytically tractable.

Faced with this problem, there are two routes that we can follow:

1) to use an analytic approximation to the integral in (1), (2), and (3) or

2) to use Monte Carlo methods, specifically MCMC methods, to approximate it.

Below, we consider an analytic approximation based on Laplace's approximation; some other approximations are discussed in Section 3.3.

In Laplace's approximation, the integrand $P\left(y_{*}, y \mid t, \boldsymbol{\theta}\right)$ is approximated by a Gaussian distribution centered at a maximum of this function with respect to $y_{*}, y$ with an inverse covariance matrix given by $-\nabla \nabla \log P\left(y_{*}, \boldsymbol{y} \mid \boldsymbol{t}, \boldsymbol{\theta}\right)$. Finding a maximum can be carried out using the NewtonRaphson iterative method on $y$, which then allows the approximate distribution of $y_{*}$ to be calculated. Details of the maximization procedure can be found in Appendix A. 


\subsection{Integration Over the Parameters}

To make predictions we integrate the predicted probabilities over the posterior $P(\boldsymbol{\theta} \mid \boldsymbol{t}) \propto P(\boldsymbol{t} \mid \boldsymbol{\theta}) P(\theta)$, as we saw in Section 2.2. For the regression problem $P(\boldsymbol{t} \mid \boldsymbol{\theta})$ can be calculated exactly using $P(\boldsymbol{t} \mid \boldsymbol{\theta})=\int P(\boldsymbol{t} \mid \boldsymbol{y}) P(\boldsymbol{y} \mid \boldsymbol{\theta}) d \boldsymbol{y}$, but this integral is not analytically tractable for the classification problem. Let $\Psi=\log P(\boldsymbol{t} \mid \boldsymbol{y})+\log P(\boldsymbol{y})$. Using $P\left(t_{i} \mid \boldsymbol{y}_{i}\right)=$ $t_{i} y_{i}-\log \left(1+e^{y_{i}}\right)$, we obtain

$$
\begin{aligned}
\Psi= & \boldsymbol{t}^{T} \boldsymbol{y}-\sum_{i=1}^{n} \log \left(1+e^{y_{i}}\right) \\
& -\frac{1}{2} \boldsymbol{y}^{T} K^{-1} \boldsymbol{y}-\frac{1}{2} \log |K|-\frac{n}{2} \log 2 \pi .
\end{aligned}
$$

By using Laplace's approximation about the maximum $\tilde{y}$ we find that

$$
\log P(\boldsymbol{t} \mid \boldsymbol{\theta}) \simeq \Psi(\widetilde{\boldsymbol{y}})-\frac{1}{2} \log \left|K^{-1}+W\right|+\frac{n}{2} \log 2 \pi .
$$

We denote the right-hand side of this equation by log $P_{a}(\boldsymbol{t} \mid \boldsymbol{\theta})$ (where $a$ stands for approximate).

The integration over $\boldsymbol{\theta}$-space also cannot be done analytically, and we employ a Markov Chain Monte Carlo method. Following Neal [15] and Williams and Rasmussen [28] we have used the Hybrid Monte Carlo (HMC) method of Duane et al [4] as described in Appendix D. We use $\log P_{a}(\boldsymbol{t} \mid \boldsymbol{\theta})$ as an approximation for $\log P(\boldsymbol{t} \mid \boldsymbol{\theta})$, and use broad Gaussian priors on the parameters.

\subsection{Previous and Related Work}

Our work on Gaussian processes for regression and classification developed from the observation in [15] that a large class of neural network models converge to GPs in the limit of an infinite number of hidden units. The computational Bayesian treatment of GPs can be easier than for neural networks. In the regression case, an infinite number of weights are effectively integrated out, and one ends up dealing only with the (hyper)parameters. Results from [17] show that Gaussian processes for regression are comparable in performance to other state-of-the-art methods.

Nonparametric methods for classification problems can be seen to arise from the combination of two different strands of work. Starting from linear regression, McCullagh and Nelder [12] developed generalized linear models (GLMs). In the two-class classification context, this gives rise to logistic regression. The other strand of work was the development of nonparametric smoothing for the regression problem. Viewed as a Gaussian process prior over functions this can be traced back at least as far as the work of Kolmogorov and Wiener in the 1940s. Gaussian process prediction is well known in the geostatistics field (see, e.g., [3]) where it is known as "kriging". Alternatively, by considering "roughness penalties" on functions, one can obtain spline methods; for recent overviews, see [25] and [8]. There is a close connection between the GP and roughness penalty views, as explored in [9]. By combining GLMs with nonparametric regression one obtains what we shall call a nonparametric GLM method for classification. Early references to this method include [21] and
[16], and discussions can also be found in texts such as [8] and [25].

There are two differences between the nonparametric GLM method as it is usually described and a Bayesian treatment. Firstly, for fixed parameters the nonparametric GLM method ignores the uncertainty in $y_{*}$ and, hence, the need to integrate over this (as described in Section 3.1).

The second difference relates to the treatment of the parameters $\boldsymbol{\theta}$. As discussed in Section 2.2, given parameters $\boldsymbol{\theta}$, one can either attempt to obtain a point estimate for the parameters or to carry out an integration over the posterior. Point estimates may be obtained by maximum likelihood estimation of $\boldsymbol{\theta}$, or by cross-validation or generalized crossvalidation (GCV) methods, see e.g., [25], [8]. One problem with CV-type methods is that if the dimension of $\boldsymbol{\theta}$ is large, then it can be computationally intensive to search over a region/grid in parameter-space looking for the parameters that maximize the criterion. In a sense, the HMC method described above is doing a similar search, but using gradient information, ${ }^{1}$ and carrying out averaging over the posterior distribution of parameters. In defense of $(\mathrm{G}) \mathrm{CV}$ methods, we note Wahba's comments (e.g., in [26], referring back to [24]) that these methods may be more robust against an unrealistic prior.

One other difference between the kinds of nonparametric GLM models usually considered and our method is the exact nature of the prior that is used. Often, the roughness penalties used are expressed in terms of a penalty on the $k$ th derivative of $y(x)$, which gives rise to a power law power spectrum for the prior on $y(x)$. There can also be differences over parameterization of the covariance function; for example it is unusual to find parameters like those for ARD introduced in (4) in nonparametric GLM models. On the other hand, Wahba et al [26] have considered a smoothing spline analysis of variance (SS-ANOVA) decomposition. In Gaussian process terms, this builds up a prior on $y$ as a sum of priors on each of the functions in the decomposition

$$
y(x)=\mu+\sum_{\alpha} y_{\alpha}\left(x_{\alpha}\right)+\sum_{\alpha, \beta} y_{\alpha \beta}\left(x_{\alpha}, x_{\beta}\right)+\ldots .
$$

The important point is that functions involving all orders of interaction (from univariate functions, which on their own give rise to an additive model) are included in this sum, up to the full interaction term which is the only one that we are using. From a Bayesian point of view, questions as to the kinds of priors that are appropriate is an interesting modeling issue.

There has also been some recent work which is related to the method presented in this paper. In Section 3.1, we mentioned that it is necessary to approximate the integral in (1), (2), and (3) and described the use of Laplace's approximation.

Following the preliminary version of this paper presented in [1], Gibbs and MacKay [7] developed an alternative analytic approximation, by using variational methods to find approximating Gaussian distributions that bound the marginal likelihood $P(\boldsymbol{t} \mid \boldsymbol{\theta})$ above and below. These

1. It would be possible to obtain derivatives of the CV-score with respect to $\boldsymbol{\theta}$, but this has not, to our knowledge, been used in practice. 
approximate distributions are then used to predict $P\left(y_{*} \mid \boldsymbol{t}, \boldsymbol{\theta}\right)$ and thus $\hat{\pi}\left(\boldsymbol{x}_{*}\right)$. For the parameters, Gibbs and MacKay estimated $\boldsymbol{\theta}$ by maximizing their lower bound on $P(\boldsymbol{t} \mid \boldsymbol{\theta})$.

It is also possible to use a fully MCMC treatment of the classification problem, as discussed in the recent paper of Neal [14]. His method carries out the integrations over the posterior distributions of $\boldsymbol{y}$ and $\boldsymbol{\theta}$ simultaneously. It works by generating samples from $P(\boldsymbol{y}, \boldsymbol{\theta} \mid \mathcal{D})$ in a two-stage process. Firstly, for fixed $\boldsymbol{\theta}$, each of the $n$ individual $y_{i}$ s are updated sequentially using Gibbs sampling. This "sweep" takes time $O\left(n^{2}\right)$ once the matrix $K^{-1}$ has been computed (in time $\left.O\left(n^{3}\right)\right)$, so it actually makes sense to perform quite a few Gibbs sampling scans between each update of the parameters, as this probably makes the Markov chain mix faster. Secondly, the parameters are updated using the Hybrid Monte Carlo method. To make predictions, one averages over the predictions made by each $\boldsymbol{y}, \boldsymbol{\theta}$ sample.

\section{GPS fOR MUltiple-Class Classification}

The extension of the preceding framework to multiple classes is essentially straightforward, although notationally more complex.

Throughout we employ a one-of- $m$ class coding scheme, ${ }^{2}$ and use the multiclass analogue of the logistic functionthe softmax function-to describe the class probabilities. The probability that an instance labeled by $i$ is in class $c$ is denoted by $\pi_{c}^{i}$, so that an upper index to denotes the example number, and a lower index the class label. Similarly, the activations associated with the probabilities are denoted by $y_{c}^{i}$. Formally, the softmax link function relates the activations and probabilities through

$$
\pi_{c}^{i}=\frac{\exp y_{c}^{i}}{\sum_{c^{\prime}} \exp y_{c^{\prime}}^{i}}
$$

which automatically enforces the constraint $\Sigma_{c} \pi_{c}^{i}=1$. The targets are similarly represented by $t_{c}^{i}$, and are specified using a one-of- $m$ coding.

The log likelihood takes the form $\mathcal{L}=\sum_{i, c} t_{c}^{i} \ln \pi_{c}^{i}$, which for the softmax link function gives

$$
\mathcal{L}=\sum_{i, c} t_{c}^{i}\left(y_{c}^{i}-\ln \sum_{c^{\prime}} \exp \pi_{c^{\prime}}^{i}\right) .
$$

As for the two class case, we shall assume that the GP prior operates in activation space; that is we specify the correlations between the activations $y_{c}^{i}$.

One important assumption we make is that our prior knowledge is restricted to correlations between the activations of a particular class. Whilst there is no difficulty in extending the framework to include interclass correlations, we have not yet encountered a situation where we felt able to specify such correlations. Formally, the activation correlations take the form,

2. That is, the class is represented by a vector of length $m$ with zero entries everywhere except for the correct component which contains 1.

$$
\left\langle y_{c}^{i} y_{c^{\prime}}^{i^{\prime}}\right\rangle=\delta_{c, c^{\prime}} K_{c}^{i, i^{\prime}}
$$

where $K_{c}^{i, i^{\prime}}$ is the $i, i^{\prime}$ element of the covariance matrix for the $c$ th class. Each individual correlation matrix $K_{c}$ has the form given by (4) for the two-class case. We shall use a separate set of parameters for each class. The use of $m$ independent processes to perform the classification is redundant, but forcing the activations of one process to be, say, zero would introduce an arbitrary asymmetry into the prior.

For simplicity, we introduce the augmented vector notation,

$$
\boldsymbol{y}_{+}=\left(y_{1}^{1}, \ldots, y_{1}^{n}, y_{1}^{*}, y_{2}^{1}, \ldots, y_{2}^{n}, y_{2}^{*}, \ldots, y_{m}^{1}, \ldots, y_{c}^{1}, \ldots, y_{m}^{*}\right)
$$

where, as in the two-class case, $y_{c}^{*}$ denotes the activation corresponding to input $x_{*}$ for class $c$; this notation is also used to define $t_{+}$and $\boldsymbol{\pi}_{+}$. In a similar manner, we define $y, t$, and $\pi$ by excluding the values corresponding to the test point $\boldsymbol{x}_{*}$. Let $\boldsymbol{y}^{*}=\left(y_{1}^{*}, y_{2}^{*}, \ldots, y_{m}^{*}\right)$.

With this definition of the augmented vectors, the GP prior takes the form

$$
P\left(\boldsymbol{y}_{+}\right) \propto \exp \left\{-\frac{1}{2} \boldsymbol{y}_{+}^{T}\left(K^{+}\right)^{-1} \boldsymbol{y}_{+}\right\},
$$

where, from (12), the covariance matrix $K^{+}$is block diagonal in the matrices, $K_{1}^{+}, \ldots, K_{m}^{+}$. Each individual matrix $K_{c}^{+}$ expresses the correlations of activations within class $c$.

As in the two-class case, to use Laplace's approximation we need to find the mode of $P\left(y_{+} \mid t\right)$. The procedure is described in Appendix C. As for the two-class case, we make predictions for $\pi\left(x^{*}\right)$ by averaging the softmax function over the Gaussian approximation to the posterior distribution of $y^{*}$. At present, we simply estimate this integral using 1,000 draws from a Gaussian random vector generator.

\section{EXPERIMENTAL RESULTS}

When using the Newton-Raphson algorithm, $\boldsymbol{\pi}$ was initialized each time with entries $1 / m$, and iterated until the mean relative difference of the elements of $W$ between consecutive iterations was less than $10^{-4}$.

For the HMC algorithm, the same step size $\varepsilon$ is used for all parameters, and should be as large as possible while keeping the rejection rate low. We have used a trajectory made up of $L=20$ leapfrog steps, which gave a low correlation between successive states. The priors over parameters were set to be Gaussian with a mean of -3 and a standard deviation of 3 . In all our simulations, a step size $\varepsilon=0.1$ produced a low rejection rate $(<5$ percent $)$. The parameters corresponding to the $w_{l}$ s were initialized to -2 and that for $v_{0}$ to 0 . The sampling procedure was run for 200 iterations, and the first third of the run was discarded; this "burn-in" is intended to give the parameters time to come close to their equilibrium distribution. Tests carried out using the R-CODA package ${ }^{3}$ on the examples in Section 5.1 suggested that this was indeed effective in removing

3. Available from the Comprehensive $\mathrm{R}$ Archive Network at http://www.ci.tuwien.ac.at. 
the transients, although we note that it is widely recognized (see, e.g., [2]) that determining when the equilibrium distribution has been reached is a difficult problem. Although the number of iterations used is much less than typically used for MCMC methods, it should be remembered that

1) each iteration involves $L=20$ leapfrog steps and

2) that by using HMC we aim to reduce the "random walk" behavior seen in methods such as the Metropolis algorithm.

Autocorrelation analysis for each parameter indicated, in general, that low correlation was obtained after a lag of a few iterations.

The MATLAB code which we used to run our experiments is available from ftp://cs.aston.ac.uk/neural/willicki/gpclass/.

\subsection{Two Classes}

We have tried out our method on two well-known twoclass classification problems, the Leptograpsus crabs and Pima Indian diabetes datasets. ${ }^{4}$ We first rescale the inputs so that they have mean of zero and unit variance on the training set. Our Matlab implementations for the HMC simulations for both tasks each take several hours on a SGI Challenge machine (200MHz R10000), although good results can be obtained in much less time. We also tried a standard Metropolis MCMC algorithm for the Crabs problem, and found similar results, although the sampling by this method is somewhat slower than that for HMC.

The results for the Crabs and Pima tasks, together with comparisons with other methods (from [20] and [18]) are given in Tables 1 and 2, respectively. The tables also include results obtained for Gaussian processes using

1) estimation of the parameters by maximizing the penalized likelihood (found using 20 iterations of a scaled conjugate gradient optimizer) and

2) Neal's MCMC method.

Details of the set-up used for Neal's method are given in Appendix E.

In the Leptograpsus crabs problem, we attempt to classify the sex of crabs on the basis of five anatomical attributes, with an optional additional color attribute. There are 50 examples available for crabs of each sex and color, making a total of 200 labeled examples. These are split into a training set of 20 crabs of each sex and color, making 80 training examples, with the other 120 examples used as the test set. The performance of our GP method is equal to the best of the other methods reported in [20], namely a two hidden unit neural network with direct input to output connections, a logistic output unit, and trained with maximum likelihood (Network(1) in Table 1). Neal's method gave a very similar level of performance. We also found that estimating the parameters using maximum penalized likelihood (MPL) gave similar performance with less than a minute of computing time.

For the Pima Indians, diabetes problem we have used the data as made available by Prof. Ripley, with his training/test split of 200 and 332 examples, respectively [18].
TABLE 1

NUMBER OF TEST ERRORS FOR THE LEPTOGRAPSUS CRABS TASK

\begin{tabular}{|c|c|c|}
\hline Method & Color given & Color not given \\
\hline Neural Network(1) & 3 & 3 \\
\hline Neural Network(2) & 5 & 3 \\
\hline Linear Discriminant & 8 & 8 \\
\hline Logistic regression & 4 & 4 \\
\hline MARS (degree =1) & 8 & 4 \\
\hline PP regression (4 ridge & 3 & 6 \\
\hline $\begin{array}{c}\text { Gaunssian Process (Laplace } \\
\text { Approximation, HMC) }\end{array}$ & 3 & 3 \\
\hline $\begin{array}{c}\text { Gaussian Process (Laplace } \\
\text { Approximation, MPL) }\end{array}$ & 4 & 3 \\
\hline $\begin{array}{c}\text { Gaussian Process (Neal's } \\
\text { method) }\end{array}$ & 4 & 3 \\
\hline
\end{tabular}

Comparisons are taken from from Ripley [18] and Ripley [20], respectively. Network(2) used two hidden units and the predictive approach (Ripley, [19]) which uses Laplace's approximation to weight each network local minimum.

The baseline error obtained by simply classifying each record as coming from a diabetic gives rise to an error of 33 percent. Again, ours and Neal's GP methods are comparable with the best alternative performance, with an error of around 20 percent, as shown in Table 2. It is encouraging that the results obtained using Laplace's approximation and Neal's method are similar. We also estimated the parameters using maximum penalized likelihood, rather than Monte Carlo integration. The performance in this case was a little worse, with 21.7 percent error, but for only two minutes computing time.

Analysis of the posterior distribution of the $w$ parameters in the covariance function (4) can be informative. Fig. 2 plots the posterior marginal mean and one standard deviation error bars for each of the seven input dimensions. Recalling that the variables are scaled to have zero mean and unit variance, it would appear that variables 1 and 3 have the shortest length scales (and therefore the most variability) associated with them.

\subsection{Multiple Classes}

Due to the rather long time taken to run our code, we were only able to test it on relatively small problems, by which we mean only a few hundred data points and several classes. Furthermore, we found that a full Bayesian integration over possible parameter settings was beyond our computational means, and we therefore had to be satisfied with a maximum penalized likelihood approach. Rather than using the potential and its gradient in a HMC routine, we now simply used them as inputs to a scaled conjugate gradient optimizer (based on [13]) instead, attempting to find a mode of the class posterior, rather than to average over the posterior distribution.

We tested the multiple-class method on the Forensic Glass dataset described in [18]. This is a dataset of 214 examples with nine inputs and six output classes. Because the

5. The performance obtained by Gibbs and MacKay in [7] was similar. Their method made four errors in the crab task (with color given), and 70 errors on the Pima dataset. 
TABLE 2

NUMBER OF TEST ERRORS ON THE PIMA INDIAN DIABETES TASK

\begin{tabular}{|c|c|}
\hline Method & Pima Indian diabetes \\
\hline Neural Network & $75+$ \\
\hline Linear Discriminant & 67 \\
\hline Logistic Regression & 66 \\
\hline MARS (degree = 1) & 75 \\
\hline PP regression (4 ridge functions) & 75 \\
\hline Gaussian Mixture & 64 \\
\hline Gaussian Process (Laplace \\
Approximation, HMC) & 68 \\
\hline Gaussian Process (Laplace & 69 \\
Approximation, MPL) & 68 \\
\hline Gaussian Process (Neal's method) & \\
\hline
\end{tabular}

Comparisons are taken from from Ripley [18] and Ripley [20], respectively. The neural network had one hidden unit and was trained with maximum likelihood; the results were worse for nets with two or more hidden units (Ripley, [18]).

dataset is so small, the performance is estimated from using 10 -fold cross validation. Computing the penalized maximum likelihood estimate of our multiple GP method took approximately 24 hours on our SGI Challenge and gave a classification error rate of 23.3 percent. As we see from Table 3, this is comparable to the best of the other methods. The performance of Neal's method is surprisingly poor; this may be due to the fact that we allow separate parameters for each of the $y$ processes, while these are constrained to be equal in Neal's code. There are also small but perhaps significant differences in the specification of the prior (see Appendix E for details).

\section{Discussion}

In this paper, we have extended the work of Williams and Rasmussen [28] to classification problems, and have demonstrated that it performs well on the datasets we have tried. We believe that the kinds of Gaussian process prior we have used are more easily interpretable than models (such as neural networks) in which the priors are on the parameterization of the function space. For example, the posterior distribution of the ARD parameters (as illustrated in Fig. 2 for the Pima Indians diabetes problem) indicates the relative importance of various inputs. This interpretability should also facilitate the incorporation of prior knowledge into new problems.

There are quite strong similarities between GP classifiers and support-vector machines (SVMs) [23]. The SVM uses a covariance kernel, but differs from the GP approach by using a different data fit term (the maximum margin), so that the optimal $y$ is found using quadratic programming. The comparison of these two algorithms is an interesting direction for future research.

A problem with methods based on GPs is that they require computations (trace, determinants and linear solutions) involving $n \times n$ matrices, where $n$ is the number of training examples, and hence run into problems on large datasets. We have looked into methods using Bayesian numerical techniques to calculate the trace and determinant [22], [6], although we found that these techniques did not work well for the (relatively) small size problems on which
TABLE 3

PERCENTAGE OF TEST ERROR FOR THE FORENSIC GLASS PROBLEM

\begin{tabular}{|c|c|}
\hline Method & Forensic Glass \\
\hline Neural Network (4HU) & $23.8 \%$ \\
\hline Linear Discriminant & $36 \%$ \\
\hline MARS (degree $=1$ ) & $32.2 \%$ \\
\hline PP regression (5 ridge functions) & $35 \%$ \\
\hline Gaussian Mixture & $30.8 \%$ \\
\hline Decision Tree & $32.2 \%$ \\
\hline Gaussian Process (LA, MPL) & $23.3 \%$ \\
\hline Gaussian Process (Neal's method) & $31.8 \%$ \\
\hline
\end{tabular}

See Ripley [18] for details of the methods.

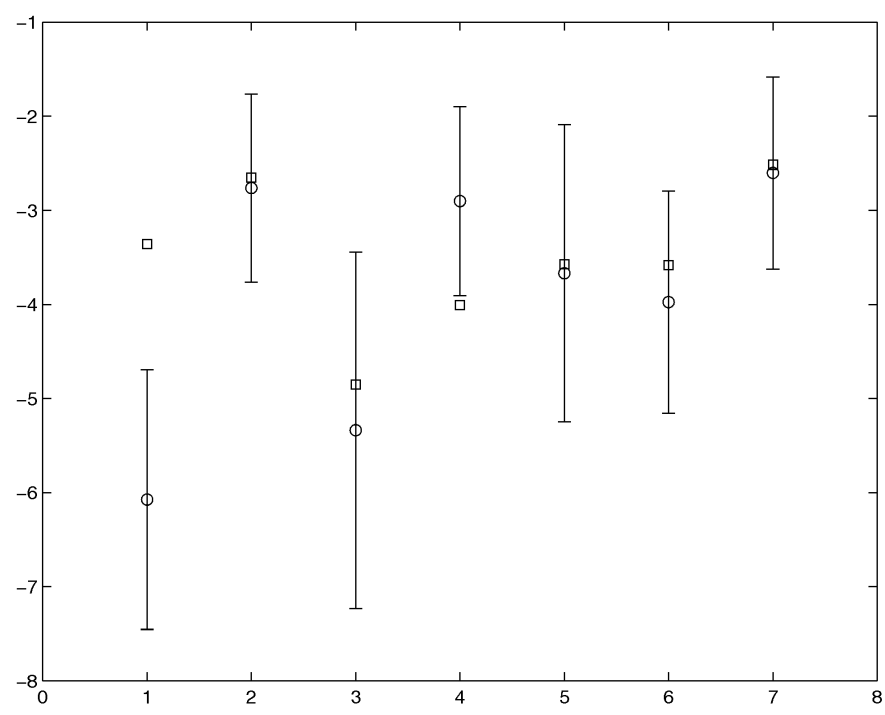

Fig. 2. Plot of the log $w$ parameters for the Pima dataset. The circle indicates the posterior marginal mean obtained from the HMC run (after burn-in), with one standard deviation error bars. The square symbol shows the log $w$-parameter values found by maximizing the penalized likelihood. The variables are: 1) the number of pregnancies, 2) plasma glucose concentration, 3) diastolic blood pressure, 4) triceps skin fold thickness, 5) body mass index, 6) diabetes pedigree function, 7) age. For comparison, Wahba et al. [26] using generalized linear regression, found that variables $1,2,5$, and 6 were the most important.

we tested our methods. Computational methods used to speed up the quadratic programming problem for SVMs may also be useful for the GP classifier problem. We are also investigating the use of different covariance functions and improvements on the approximations employed.

\section{APPENDIX A \\ MAXIMIZING $P\left(\boldsymbol{y}_{+} \mid \boldsymbol{t}\right)$ : Two-CLASS CASE}

We describe how to find iteratively the vector $\boldsymbol{y}_{+}$so that $P\left(y_{+} \mid t\right)$ is maximized. This material is also covered in [8, Section 5.3.3] and [25, Section 9.2]. We provide it here for completeness and so that the terms in (9) are well-defined.

Let $\boldsymbol{y}_{+}$denote $\left(y_{*}, \boldsymbol{y}\right)$, the complete set of activations. By Bayes' theorem $\log P\left(\boldsymbol{y}_{+} \mid \boldsymbol{t}\right)=\log P(\boldsymbol{t} \mid \boldsymbol{y})+\log P\left(\boldsymbol{y}_{+}\right)-\log P(\boldsymbol{t})$, and let $\Psi_{+}=\log P(\boldsymbol{t} \mid \boldsymbol{y})+\log P\left(\boldsymbol{y}_{+}\right)$. As $P(\boldsymbol{t})$ does not depend on $y_{+}$(it is just a normalizing factor), the maximum of 
$P\left(y_{+} \mid \boldsymbol{t}\right)$ is found by maximizing $\Psi_{+}$with respect to $y_{+}$. Using $\log P\left(t_{i} \mid y_{i}\right)=t_{i} y_{i}-\log \left(1+e^{y_{i}}\right)$, we obtain

$$
\begin{aligned}
\Psi_{+}= & \boldsymbol{t}^{T} \boldsymbol{y}-\sum_{i=1}^{n} \log \left(1+e^{y_{i}}\right)-\frac{1}{2} \boldsymbol{y}_{+}^{T} K_{+}^{-1} \boldsymbol{y}_{+} \\
& -\frac{1}{2} \log \left|K_{+}\right|-\frac{n+1}{2} \log 2 \pi,
\end{aligned}
$$

where $K_{+}$is the covariance matrix of the GP evaluated at $x_{1}, \ldots x_{n}, x_{*} . \Psi$ is defined similarly in (8). $K_{+}$can be partitioned in terms of an $n \times n$ matrix $K$, a $n \times 1$ vector $k$ and a scalar $k_{*}$ viz.

$$
K_{+}=\left(\begin{array}{cc}
K & \boldsymbol{k} \\
\boldsymbol{k}^{T} & k_{*}
\end{array}\right) .
$$

As $y_{*}$ only enters into (14) in the quadratic prior term and has no data point associated with it, maximizing $\Psi_{+}$with respect to $y_{+}$can be achieved by first maximizing $\Psi$ with respect to $y$ and then doing the further quadratic optimization to determine $y_{*}$. To find a maximum of $\Psi$, we use the Newton-Raphson iteration $y^{\text {new }}=y-(\nabla \nabla \Psi)^{-1} \nabla \Psi$. Differentiating (8) with respect to $y$ we find

$$
\begin{aligned}
\nabla \Psi & =(\boldsymbol{t}-\boldsymbol{\pi})-K^{-1} \boldsymbol{y} \\
\nabla \nabla \Psi & =-K^{-1}-W,
\end{aligned}
$$

where the "noise" matrix is given by $W=\operatorname{diag}\left(\pi_{1}\left(1-\pi_{1}\right), .\right.$. $\left.\pi_{n}\left(1-\pi_{n}\right)\right)$. This results in the iterative equation,

$$
y^{\text {new }}=\left(K^{-1}+W\right)^{-1} W\left(y+W^{-1}(\boldsymbol{t}-\boldsymbol{\pi})\right) .
$$

To avoid unnecessary inversions, it is usually more convenient to rewrite this in the form

$$
\boldsymbol{y}^{\text {new }}=K(I+W K)^{-1}(W \boldsymbol{y}+(\boldsymbol{t}-\boldsymbol{\pi})) \text {. }
$$

Note that $-\nabla \nabla \Psi$ is always positive definite, so that the optimization problem is convex.

Given a converged solution $\tilde{y}$ for $y, y_{*}$ can easily be found using $y_{*}=\boldsymbol{k}^{T} K^{-1} \tilde{\boldsymbol{y}}=\boldsymbol{k}^{T}(\boldsymbol{t}-\tilde{\boldsymbol{\pi}})$, as $K^{-1} \tilde{\boldsymbol{y}}=(\boldsymbol{t}-\tilde{\boldsymbol{\pi}})$ from (16). $\operatorname{var}\left(y_{*}\right)$ is given by $\left(K_{+}^{-1}+W_{+}\right)_{(n+1)(n+1)}^{-1}$, where $W_{+}$ is the $W$ with a zero appended in the $(n+1)$ th diagonal position. Given the mean and variance of $y_{*}$ it is then easy to find $\hat{\pi}_{*}=\int \pi_{*} P\left(\pi_{*} \mid t\right) d \pi_{*}$, the mean of the distribution of $P\left(\pi_{*} \mid \boldsymbol{t}\right)$. In order to calculate the Gaussian integral over the logistic sigmoid function, we employ an approximation based on the expansion of the sigmoid function in terms of the error function. As the Gaussian integral of an error function is another error function, this approximation is fast to compute. Specifically, we use a basis set of five scaled error functions to interpolate the logistic sigmoid at chosen points. ${ }^{6}$ This gives an accurate approximation (to $10^{-4}$ ) to the desired integral with a small computational cost.

The justification of Laplace's approximation in our case is somewhat different from the argument usually put forward, e.g., for asymptotic normality of the maximum

6. In detail, we used the basis functions $\operatorname{erf}(\lambda x))$ for $\lambda=[0.41,0.4,0.37$, $0.44,0.39]$. These were used to interpolate $\sigma(x)$ at $x=[0,0.6,2,3.5,4.5, \infty]$. likelihood estimator for a model with a finite number of parameters. This is because the dimension of the problem grows with the number of data points. However, if we consider the "infill asymptotics" (see, e.g., [3]), where the number of data points in a bounded region increases, then a local average of the training data at any point $x$ will provide a tightly localized estimate for $\pi(x)$ and hence $y(x)$ (this reasoning parallels more formal arguments found in [29]). Thus, we would expect the distribution $P(y)$ to become more Gaussian with increasing data.

\section{APPENDIX B \\ DeRivatives of $\log P_{a}(\boldsymbol{t} \mid \boldsymbol{\theta})$ wrt $\boldsymbol{\theta}$}

For both the HMC and MPL methods, we require the derivative of $l_{a}=\log P_{a}(\boldsymbol{t} \mid \boldsymbol{\theta})$ with respect to components of $\boldsymbol{\theta}$, for example $\theta_{k}$. This derivative will involve two terms, one due to explicit dependencies of

$$
l_{a}=\Psi(\widetilde{y})-\frac{1}{2} \log \left|K^{-1}+W\right|+\frac{n}{2} \log 2 \pi
$$

on $\theta_{k}$, and also because a change in $\boldsymbol{\theta}$ will cause a change in $\tilde{y}$. However, as $\tilde{y}$ is chosen so that $\left.\nabla \Psi(y)\right|_{y=\tilde{y}}=0$, we obtain

$$
\frac{\partial l_{a}}{\partial \theta_{k}}=\left.\frac{\partial l_{a}}{\partial \theta_{k}}\right|_{\text {explicit }}-\frac{1}{2} \sum_{i=1}^{n} \frac{\partial \log \left|K^{-1}+W\right|}{\partial \widetilde{y}_{i}} \frac{\partial \tilde{y}_{i}}{\partial \theta_{k}} .
$$

The dependence of $\left|K^{-1}+W\right|$ on $\tilde{y}$ arises through the dependence of $W$ on $\tilde{\boldsymbol{\pi}}$, and, hence, $\tilde{\boldsymbol{y}}$. By differentiating $\widetilde{y}=K(t-\tilde{\pi})$, one obtains

$$
\frac{\partial \tilde{y}}{\partial \theta_{k}}=(I+K W)^{-1} \frac{\partial K}{\partial \theta_{k}}(\boldsymbol{t}-\tilde{\boldsymbol{\pi}}),
$$

and, hence, the required derivative can be calculated.

\section{APPENDIX C \\ Maximizing $P\left(\boldsymbol{y}_{+} \mid \boldsymbol{t}\right)$ : Multiple-Class Case}

The GP prior and likelihood, defined by (13) and (11), define the posterior distribution of activations, $P\left(y_{+} \mid \boldsymbol{t}\right)$. As in Appendix A we are interested in a Laplace approximation to this posterior, and therefore need to find the mode with respect to $y_{+}$. Dropping unnecessary constants, the multiclass analogue of (14) is

$$
\Psi_{+}=-\frac{1}{2} y_{+}^{T} K_{+}^{-1} y_{+}-\frac{1}{2} \log \left|K_{+}\right|+\boldsymbol{t}^{T} \boldsymbol{y}-\sum_{i} \ln \sum_{c} \exp y_{c}^{i} .
$$

By the same principle as in Appendix A, we define $\Psi$ by analogy with (8), and first optimize $\Psi$ with respect to $y$, afterwards performing the quadratic optimization of $\Psi_{+}$ with respect to $y_{*}$.

In order to optimize $\Psi$ with respect to $y$, we make use of the Hessian given by

$$
\nabla \nabla \Psi=-K^{-1}-W,
$$

where $K$ is the $m n \times m n$ block-diagonal matrix with blocks $K_{c}, c=1, \ldots, m$. Although this is in the same form as for the two-class case, (17), there is a slight change in the definition of the "noise" matrix, $W$. A convenient way to define $W$ is 
by introducing the matrix $\Pi$ which is a $m n \times n$ matrix of the form $\Pi=\left(\operatorname{diag}\left(\pi_{1}^{1} . . \pi_{1}^{n}\right), \ldots, \operatorname{diag}\left(\pi_{m}^{1} . . \pi_{m}^{n}\right)\right)$. Using this notation, we can write the noise matrix in the form of a diagonal matrix and an outer product,

$$
W=-\operatorname{diag}\left(\pi_{1}^{1} . . \pi_{1}^{n}, \ldots, \pi_{m}^{1} . . \pi_{m}^{n}\right)+\Pi \Pi^{T} .
$$

As in the two-class case, we note that $-\nabla \nabla \Psi$ is again positive definite, so that the optimization problem is convex.

The update equation for iterative optimization of $\Psi$ with respect to the activations $y$ then follows the same form as that given by (18). The advantage of the representation of the noise matrix in (23) is that we can then invert matrices and find their determinants using the identities,

$$
\left(A+\Pi^{T}\right)^{-1}=A^{-1}-A^{-1} \Pi\left(I_{n}+\Pi^{T} A^{-1} \Pi\right)^{-1} \Pi^{T} A^{-1}
$$

and

$$
\operatorname{det}\left(A+\Pi \Pi^{T}\right)=\operatorname{det}(A) \operatorname{det}\left(I_{n}+\Pi^{T} A^{-1} \Pi\right),
$$

where $A=K^{-1}+\operatorname{diag}\left(\pi_{1}^{1} . . \pi_{m}^{n}\right)$. As $A$ is block-diagonal, it can be inverted blockwise. Thus, rather than requiring determinants and inverses of a $m n \times m n$ matrix, we only need to carry out expensive matrix computations on $n \times$ $n$ matrices. The resulting update equations for $y$ are then of the same form as given in (18), where the noise matrix and covariance matrices are now in their multiple class form.

Essentially, these are all the results needed to generalize the method to the multiple-class problem. Although, as we mentioned above, the time complexity of the problem does not scale with the $m^{3}$, but rather $m$ (due to the identities in (24), (25)), calculating the function and its gradient is still rather expensive. We have experimented with several methods of mode finding for the Laplace approximation. The advantage of the Newton iteration method is its fast quadratic convergence. An integral part of each Newton step is the calculation of the inverse of a matrix $M$ acting upon a vector, i.e., $M^{-1} b$. In order to speed up this particular step, we used a conjugate gradient (CG) method to solve iteratively the corresponding linear system $\mathrm{Mz}=\boldsymbol{b}$. As we repeatedly need to solve the system (because $W$ changes as $y$ is updated), it saves time not to run the CG method to convergence each time it is called. In our experiments, the CG algorithm was terminated when $1 / n \sum_{i=1}^{n}\left|r_{i}\right|<10^{-9}$, where $\boldsymbol{r}=\mathrm{Mz}-\boldsymbol{b}$.

The calculation of the derivative of $\log P_{a}(\boldsymbol{t} \mid \boldsymbol{\theta})$ wrt $\boldsymbol{\theta}$ in the multiple-class case is analogous to the two-class case described in Appendix B.

\section{APPENDIX D \\ Hybrid Monte Carlo}

HMC works by creating a fictitious dynamical system in which the parameters are regarded as position variables, and augmenting these with momentum variables $p$. The purpose of the dynamical system is to give the parameters "inertia" so that random-walk behavior in $\boldsymbol{\theta}$-space can be avoided. The total energy, $H$, of the system is the sum of the kinetic energy, $K=p^{T} p / 2$ and the potential energy, $E$. The potential energy is defined such that $p(\boldsymbol{\theta} \mid D) \propto \exp (-E)$, i.e., $E=-\log P(\boldsymbol{t} \mid \boldsymbol{\theta})-\log P(\boldsymbol{\theta})$. We sample from the joint distribution for $\boldsymbol{\theta}$ and $\boldsymbol{p}$ given by $P(\boldsymbol{\theta}, \boldsymbol{p}) \propto \exp (-E-K)$; the marginal of this distribution for $\boldsymbol{\theta}$ is the required posterior. A sample of parameters from the posterior can therefore be obtained by simply ignoring the momenta.

Sampling from the joint distribution is achieved by two steps:

1) finding new points in phase space with near-identical energies $H$ by simulating the dynamical system using a discretised approximation to Hamiltonian dynamics and

2) changing the energy $H$ by Gibbs sampling the momentum variables.

Hamilton's first-order differential equations for $H$ are approximated using the leapfrog method which requires the derivatives of $E$ with respect to $\boldsymbol{\theta}$. Given a Gaussian prior on $\boldsymbol{\theta}, \log P(\boldsymbol{\theta})$ is straightforward to differentiate. The derivative of $\log P_{a}(\boldsymbol{t} \mid \boldsymbol{\theta})$ is also straightforward, although implicit dependencies of $\tilde{y}$ (and, hence, $\tilde{\pi}$ ) on $\boldsymbol{\theta}$ must be taken into account as described in Appendix B. The calculation of the energy can be quite expensive as for each new $\boldsymbol{\theta}$, we need to perform the maximization required for Laplace's approximation, (9). This proposed state is then accepted or rejected using the Metropolis rule depending on the final energy $H^{*}$ (which is not necessarily equal to the initial energy $H$ because of the discretization).

\section{APPENDIX E \\ Simulation Set-Up for Neal's Code}

We used the fbm software available from http://www.cs.utoronto. $\mathrm{ca} / \mathrm{radford} / \mathrm{fbm}$.software.html. For example, the commands used to run the Pima example are

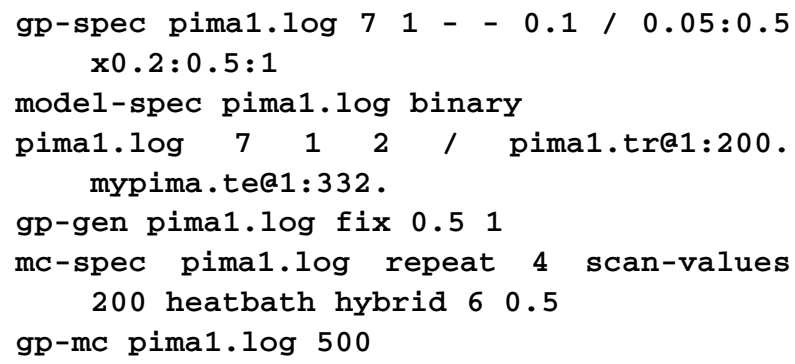

which follow closely the example given in Neal's documentation.

The gp-spec command specifies the form of the Gaussian process, and in particular the priors on the parameters $v_{0}$ and the $w$ s (see (4)). The expression $0.05: 0.5$ specifies a Gamma-distribution prior on $v_{0}$, and $\mathbf{x} 0.2: 0.5: 1$ specifies a hierarchical Gamma prior on the ws. Note that a "jitter" of 0.1 is also specified on the prior covariance function; this improves conditioning of the covariance matrix.

The mc-spec command gives details of the MCMC updating procedure. It specifies four repetitions of 200 scans of the $y$ values followed by six HMC updates of the parameters (using a step-size adjustment factor of 0.5). gp-mc specifies that this is sequence is carried out 500 times. 
We aimed for a rejection rate of around 5 percent. If this was exceeded, the stepsize reduction factor was reduced and the simulation run again.

\section{ACKNOWLEDGMENTS}

We thank Prof. B. Ripley for making available the Leptograpsus crabs, Pima Indian diabetes, and Forensic Glass datasets. This work was partially supported by EPSRC grant GR/J75425, Novel Developments in Learning Theory for Neural Networks, and much of the work was carried out at Aston University. The authors gratefully acknowledge the hospitality provided by the Isaac Newton Institute for Mathematical Sciences (Cambridge, UK) where this paper was written. We thank Mark Gibbs, David MacKay, and Radford Neal for helpful discussions, and the anonymous referees for their comments which helped improve the paper.

\section{REFERENCES}

[1] D. Barber and C.K.I Williams, "Gaussian Processes for Bayesian Classification via Hybrid Monte Carlo," M.C. Mozer, M.I. Jordan, and T. Petsche, eds., Advances in Neural Information Processing Systems 9. MIT Press, 1997.

[2] M.K. Cowles and B.P. Carlin, "Markov-Chain Monte-Carlo Convergence Diagnostics-A Comparative Review," J. Am. Statistics Assoc., vol. 91, pp. 883-904, 1996.

[3] N.A.C. Cressie, Statistics for Spatial Data. New York, NY: Wiley, 1993.

[4] S. Duane, A.D. Kennedy, B.J. Pendleton, and D. Roweth, "Hybrid Monte Carlo," Physics Letters B, vol. 195, pp. 216-222, 1987.

[5] A. Gelman, J.B. Carlin, H.S. Stern, and D.B. Rubin, Bayesian Data Analysis. London: Chapman and Hall, 1995.

[6] M. Gibbs and D.J.C. MacKay, "Efficient Implementation of Gaussian Processes," Draft manuscript, available from http://wol.raphy. cam.ac.uk/mackay/homepage.html., 1997.

[7] M. Gibbs and D.J.C. MacKay, "Variational Gaussian Process Classifiers," Draft manuscript, available via http://wol.raphy.cam.ac.uk mackay/homepage.html., 1997.

[8] P. J. Green and B. W. Silverman, Nonparametric Regression and Generalized Linear Models. London: Chapman and Hall, 1994

[9] G. Kimeldorf and G. Wahba, "A Correspondence Between Bayesian Estimation of Stochastic Processes and Smoothing by Splines," Annals of Math. Statistics, vol. 41, pp. 495-502, 1970.

[10] D.J.C. MacKay, "Bayesian Methods for Backpropagation Networks," J.L. van Hemmen, E. Domany, and K. Schulten, eds., Models of Neural Networks II. Springer, 1993.

[11] K.V. Mardia and R.J. Marshall, "Maximum Likelihood Estimation for Models of Residual Covariance in Spatial Regression," Biometrika, vol. 71, no. 1, pp. 135-146, 1984.

[12] P. McCullagh, and J. Nelder, Generalized Linear Models. Chapman and Hall, 1983

[13] M. Møller, "A Scaled Conjugate Gradient Algorithm for Fast Supervised Learning," Neural Networks, vol. 6, no. 4, pp. 525-533, 1993.

[14] R.M. Neal, "Monte Carlo Implementation of Gaussian Process Models for Bayesian Regression and Classification," Technical Report 9702, Dept. of Statistics, Univ. of Toronto, 1997. Available from http://www.cs.toronto.edu/ radford/.

[15] R.M. Neal, Bayesian Learning for Neural Networks. New York Springer, 1996. Lecture Notes in Statistics 118.

[16] F. O'Sullivan, B.S. Yandell, and W.J. Raynor, "Automatic Smoothing of Regression Functions in Generalized Linear Models," J. Am. Statistical Assoc., vol. 81, pp. 96-103, 1986.

[17] C.E. Rasmussen, Evaluation of Gaussian Processes and Other Methods for Non-Linear Regression. PhD thesis, Dept. of Computer Science, Univ. of Toronto, 1996. Available from http://www.cs.utoronto.ca/ carl/.

[18] B. Ripley, Pattern Recognition and Neural Networks. Cambridge, UK Cambridge Univ. Press, 1996.
[19] B.D. Ripley, "Statistical Aspects of Neural Networks," O.E. Barndorff-Nielsen, J.L. Jensen, and W.S. Kendall, eds., Networks and Chaos-Statistical and Probabilistic Aspects, pp. 40-123. Chapman and Hall, 1993.

[20] B.D. Ripley, "Flexible Non-Linear Approaches to Classification," V. Cherkassy, J.H. Friedman, and H. Wechsler, eds., From Statistics to Neural Networks, pp. 105-126. Springer, 1994.

[21] B.W. Silverman, "Density Ratios, Empirical Likelihood and Cot Death," Applied Statistics, vol. 27, no. 1, pp. 26-33, 1978.

[22] J. Skilling, "Bayesian Numerical Analysis," W.T. Grandy, Jr. and P. Milonni, eds., Physics and Probability. Cambridge Univ. Press, 1993.

[23] V.N. Vapnik, The Nature of Statistical Learning Theory. New York, NY: Springer Verlag, 1995.

[24] G. Wahba, "A Comparison of GCV and GML for Choosing the Smoothing Parameter in the Generalized Spline Smoothing Problem," Annals of Statistics, vol. 13, pp. 1,378-1,402, 1985.

[25] G. Wahba, Spline Models for Observational Data. Soc. Industrial and Applied Mathematics, 1990. CBMS-NSF Regional Conf. Series in Applied Mathematics.

[26] G. Wahba, C. Gu, Y. Wang, and R. Chappell, "Soft Classification, a.k.a., Risk Estimation, via Penalized Log Likelihood and Smoothing Spline Analysis of Variance," D.H. Wolpert, ed., The Mathematics of Generalization. Addison-Wesley, 1995. Proc. vol. XX in the Santa Fe Institute Studies in the Sciences of Complexity.

[27] C.K.I. Williams, "Computing With Infinite Networks," M.C. Mozer, M.I. Jordan, and T. Petsche, eds., Advances in Neural Information Processing Systems 9. MIT Press, 1997.

[28] C.K.I. Williams and C.E. Rasmussen, "Gaussian Processes for Regression," D.S. Touretzky, M.C. Mozer, and M.E. Hasselmo, eds., Advances in Neural Information Processing Systems 8, pp. 514520. MIT Press, 1996.

[29] S.J. Yakowitz and F. Szidarovszky, "A Comparison of Kriging With Nonparametric Regression Methods," J. Multivariate Analysis, vol. 16, pp. 21-53, 1985.

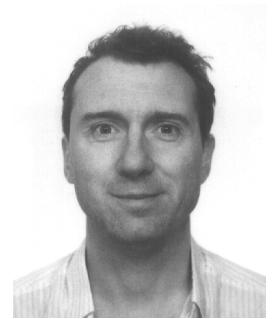

Chris Williams studied physics at Cambridge, graduating in 1982 and continued on to do Part III Maths (1983). He then received an MSc in water resources at the University of Newcastle upon Tyne before going to work in Lesotho, Southern Africa in low-cost sanitation. In 1988, he returned to academia, studying neural networks/artificial intelligence with Geoff Hinton at the University of Toronto (MSc 1990, PhD 1994). $\mathrm{He}$ was a member of the Neural Computing Research Group at Aston University from 1994 to 1998 , and is currently a lecturer in the Division of Informatics at the University of Edinburgh.

His research interests cover a wide range of theoretical and practical issues in neural networks, statistical pattern recognition, computer vision, and artificial intelligence.

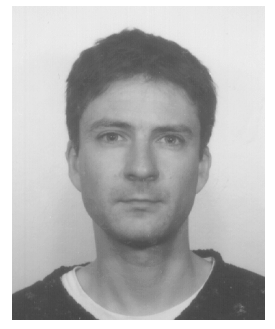

David Barber completed the University of Cambridge mathematics tripos in 1990. Following a year at Heidelberg University, Germany, he received an MSc in neural networks at King's College, London. Subsequently, he went to the University of Edinburgh, completing a $\mathrm{PhD}$ in the theoretical physics department with a study of the statistical mechanics of machine learning. After a two-year research position at Aston University, he took up his current research position at the University of Nijmegen, Holland. His main interests include machine learning, Bayesian techniques, and statistical mechanics. 